Journal of

Molecular Microbiology

and Biotechnology
J Mol Microbiol Biotechnol 2013;23:308-320

DOI: $10.1159 / 000351343$

Published online: August 5, 2013

\title{
Eut Bacterial Microcompartments: \\ Insights into Their Function, Structure, and Bioengineering Applications
}

\author{
Mark Held Maureen B. Quin Claudia Schmidt-Dannert \\ Department of Biochemistry, Molecular Biology and Biophysics, University of Minnesota, St. Paul, Minn., USA
}

\section{Key Words}

Bacterial microcompartments - Ethanolamine utilization .

Protein shell · Protein complexes · Enzyme targeting ·

Compartment phylogeny

\begin{abstract}
Bacterial microcompartments (BMCs) are protein-based polyhedral organelles which serve to encapsulate and organize enzymes involved in key metabolic pathways. The sequestration of these pathways not only improves the overall reaction efficiency; it can also harbor toxic or volatile pathway intermediates, which would otherwise be detrimental to the cell. Genomic and phylogenetic analyses reveal the presence of these unique organelles in a diverse range of bacterial species, highlighting their evolutionary importance and the essential role that they play in bacterial cell survival. Functional and structural analyses of BMCs involved in ethanolamine utilization are developing our understanding of the self-assembly and encapsulation mechanisms employed by these protein supercomplexes. This knowledge will open up exciting new avenues of research with a range of potential engineering and biotechnological applications.
\end{abstract}

Copyright $\odot 2013$ S. Karger AG, Basel

M.H. and M.B.Q. contributed equally to this study.

\section{Introduction}

It has become increasingly evident that the cytosol of bacterial cells is highly spatially organized, with an impressive array of subcellular architectural assemblies already described in the literature [Gitai, 2005]. One such organizational element, which apparently plays an essential role in the encapsulation of key metabolic processes, is the bacterial microcompartment (BMC). BMCs are proteinaceous organelles, the outer shell of which is formed by a single layer of thousands of copies of regular repeating protein arrays. The unique properties of these capsid-like assemblies allow a diverse range of bacteria to encapsulate various metabolic pathways within the lumen of the shell. By encapsulating these processes, cells improve the reaction efficiency by spatially ordering pathway enzymes in close proximity with metabolic partners, and their substrates and cofactors. Encapsulation also provides a means by which the cell can sequester reaction intermediates which may otherwise be toxic to the host. Meanwhile, passage of particular substrates to the interior and certain metabolites to the exterior of the compartment is believed to occur by a process of diffusion through pores which punctuate the shell, thereby allowing a continuous reaction scheme to occur [Bobik, 2006; Cheng et al., 2008].

Claudia Schmidt-Dannert

Department of Biochemistry, Molecular Biology and Biophysics

University of Minnesota

St. Paul, MN 55108 (USA)

E-Mail schmi232@umn.edu 
The first insights into the role that BMCs play were gained by studies into the carboxysome, a protein polyhedral assembly that is found in Cyanobacteria and chemoautotrophs, and is widely accepted as the paradigm for BMC form and function [Kerfeld et al., 2010; Shively et al., 1973b; Yeates et al., 2008]. Initially described over 50 years ago as cellular inclusions [Drews and Niklowitz, 1956], these polyhedral structures were first isolated and characterized almost 20 years later [Shively et al., 1973a, b] from the chemoautotroph Halothiobacillus neapolitanus. Early physiological and genetic studies revealed that certain microorganisms are able to grow under inorganic carbon-limiting conditions due to the fact that the carboxysome encapsulates both carbonic anhydrase and the inherently inefficient Rubisco, thereby allowing the microbe to fix carbon dioxide more effectively via what became known as a carbon concentrating-mechanism [Cannon et al., 1991]. Subsequently, studies have been undertaken to elucidate the structure of carboxysomes, to deduce not only their overall architecture, but also to understand the mechanisms by which the outer shells form. Electron microscopy studies of purified carboxysomes revealed polyhedral bodies with a diameter of $100 \mathrm{~nm}$ [Shively et al., 1973a], and that these bodies appeared to be icosahedral, displaying 5-, 3- and 2-fold symmetry about 20 triangular faces [Schmid et al., 2006]. Further insight into the formation of these bodies has been provided by solving the crystal structures of several of the proteins which integrate as the outer shell of the carboxysome. These structures illustrated that some of the proteins that form the shell $(\mathrm{CcmK} 1, \mathrm{CcmK} 2$ and $\mathrm{CcmK} 4$ of the $\beta$-carboxysome; Csos1A, Csos1D, Csos1C of the $\alpha$-carboxysome) share a conserved protein fold, which has since been named the BMC domain (Pf00936) [Kerfeld et al., 2005; Klein et al., 2009; Tanaka et al., 2008, 2009; Tsai et al., 2007].

With the increasing availability of genome sequence data, it has become apparent that the BMC domain is not limited to the genomic context of the carboxysome, but can also be found in operons encoding other protein assemblies in a diverse range of bacteria. The BMC domain has also been identified and studied in enteric bacteria such as Escherichia coli, Clostridium difficile and Salmonella enterica; pathogenic microbes that produce BMCs. These pathogens maintain operons that encode for BMC domain proteins enabling them to form large protein complexes. These protein structures serve to encapsulate metabolic enzymes that permit microbial growth and survival on substrates such as 1,2-propanediol ( $p d u)$, ethanol (etu) and ethanolamine (eut) [Bobik et al., 1999; Chen et al., 1994; Havemann and Bobik, 2003; Heldt et al., 2009; Kofoid et al., 1999; Penrod and Roth, 2006]. Electron micrographs of purified propanediol utilization (Pdu) and ethanolamine utilization (Eut) microcompartments have shown that these protein assemblies appear somewhat less symmetrical than carboxysomes [Choudhary et al., 2012; Havemann and Bobik, 2003]. Nevertheless, crystal structures of several of the Pdu and Eut proteins believed to be involved in shell assembly indicated that they share the conserved BMC domain [Crowley et al., 2008, 2010; Tanaka et al., 2010], further implicating this protein fold as a ubiquitous constituent of protein assemblies required for environmental adaption in a diverse range of bacteria. Many of the structural and functional features of carboxysomes and Pdu BMCs have been addressed in excellent reviews elsewhere [Bobik, 2006; Cannon et al., 2001; Kerfeld et al., 2010; Yeates et al., 2008, 2010, 2011], therefore for the purpose of this review, emphasis will be on the paralogous Eut BMCs.

\section{Functional Aspects of Eut BMCs}

\section{The eut Operon}

Ethanolamine is formed by the breakdown of the intestinal cell wall membrane component phosphatidylethanolamine. A number of intestinal-associated bacteria, such as Enterococcus spp., Clostridium spp. and Salmonella spp. can use ethanolamine as a sole carbon or nitrogen source [Garsin, 2010]. The genes encoding the enzymes required for ethanolamine utilization are clustered on the eut operon; which in the case of S. enterica LT2 consists of a $>15-\mathrm{kb}$ operon composed of 17 open reading frames; along with genes encoding BMC shell proteins (fig. 1) [Kofoid et al., 1999; Roof and Roth, 1988, 1989]. The formation of Eut BMCs is believed to play an essential role in shielding the cell from the volatile ethanolamine breakdown intermediate, acetaldehyde, and to prevent unnecessary loss of this potential source of carbon [Penrod and Roth, 2006]. While the exact nature of the metabolic role that Eut BMCs play is yet to be elucidated, detailed mutational, biochemical and physiological studies have provided sufficient evidence for a model of the encapsulated enzymatic pathways to be proposed, depicted schematically in figure 1 , and presented in detail below.

\section{Functional Models for the eut Operon}

The outer proteinaceous barrier formed by the shell of the Eut BMC is composed of regular repeating patterns of 


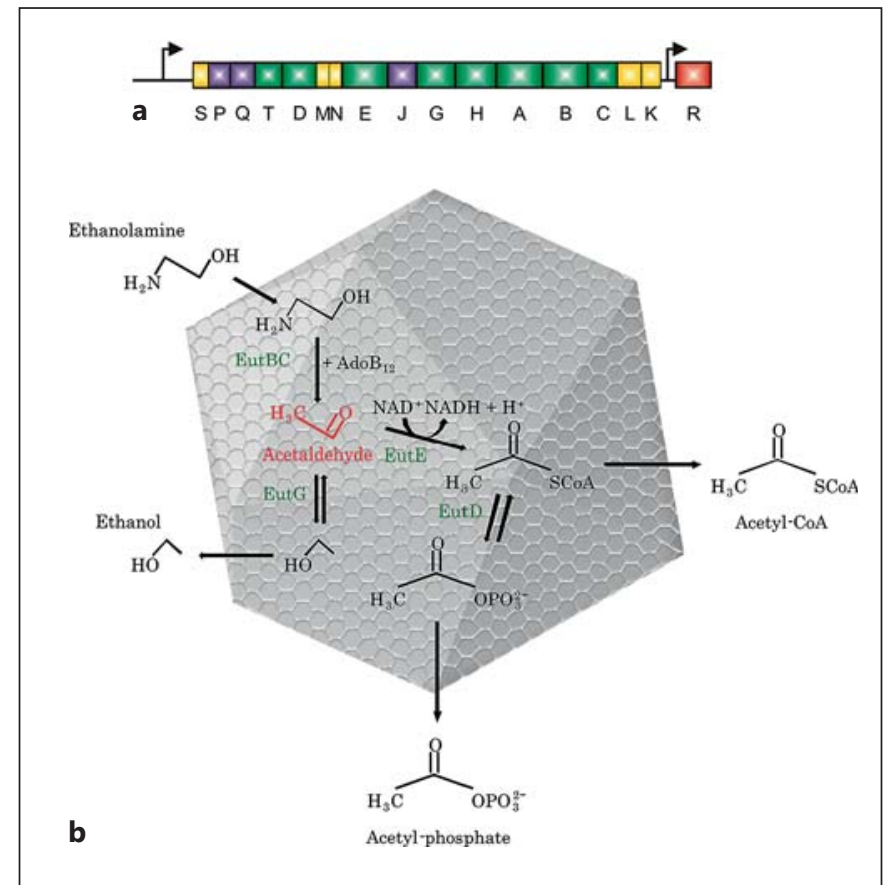

Fig. 1. Eut operon structure and function. a A schematic representation of the eut operon from S. enterica LT2. Promoters are represented as black arrows. Genes are named according to the proteins that they encode. Genes encoding regulatory elements are colored orange, those encoding shell proteins are colored yellow, genes encoding metabolic enzymes or helpers/chaperones are colored green. Those that are colored purple have no assigned function. $\mathbf{b}$ A model of the breakdown of ethanolamine within the Eut BMC. Ethanolamine enters the BMC lumen and is metabolized to ethanol, acetyl-phosphate and acetyl-CoA. Encapsulation within the Eut BMC prevents the volatile reaction intermediate acetaldehyde, colored red, from entering the cytosol of the cell. Metabolic enzymes encapsulated within the BMC lumen include ethanolamine ammonia lyase (EutBC), alcohol dehydrogenase (EutG), aldehyde dehydrogenase (EutE), and phosphotransacetylase (EutD), all colored green.

the BMC domain proteins encoded by eutS, $M, L$ and $K$, and may also include the non-BMC domain protein encoded by eutN [Kofoid et al., 1999]. Ethanolamine enters the lumen of the Eut microcompartment by passage through pores which punctate the outer shell of the BMC, either by a process of diffusion or via a $\mathrm{pH}$-sensitive facilitator encoded by eutH [Penrod et al., 2004]. Within the lumen of the BMC, ethanolamine is broken down to acetaldehyde and ammonia by the encapsulated ethanolamine ammonia lyase encoded by eut BC [Roof and Roth, 1989]; an enzyme complex which requires both the cofactor adenosylcobalamin (AboCbl) produced by a corrinoid cobalamin adenosyltransferase encoded by eut $T$
[Buan et al., 2004; Sheppard et al., 2004], and the reactivating factor encoded by eutA [Mori et al., 2004]. The resulting acetaldehyde may be converted to acetyl-CoA by a dehydrogenase encoded by eutE, either to be used directly in the TCA cycle, or to be converted to acetylphosphate by EutD and subsequently used for ATP generation [Brinsmade and Escalante-Semerena, 2004]. Alternatively, the alcohol dehydrogenase encoded by eut $G$ may convert the acetaldehyde to ethanol in order to prevent unnecessary buildup of this toxic intermediate [Stojiljkovic et al., 1995].

Finally, the purpose of some of the genes in the eut operon remains to be elucidated, namely eut $J$, eutP and eutQ. EutJ shares sequence similarity with the Hsp70 and DnaK chaperones and may therefore be involved in acting as a chaperone for the EutBC enzyme complex; however, this hypothesis is speculative [Kofoid et al., 1999; Stojiljkovic et al., 1995]. Neither EutP nor EutQ have been functionally characterized, although EutP has been annotated as a putative GTPase, and EutQ as an Eut protein [Tsoy et al., 2009]. Experimental evidence to support these annotations is yet to be presented. A recent crystal structure of EutQ from Clostridium difficile revealed a modified cupin domain. However, the enzymatic function of this protein is yet to be determined [Pitts et al., 2012].

\section{Regulation of the eut Operon}

Detailed mutational analyses have provided a partial understanding of the regulation of the Eut pathway in Salmonella. An inducible primary promoter $\left(\mathrm{P}_{\mathrm{I}}\right)$ is found at the start of the operon, upstream of eutS. A second promoter $\left(\mathrm{P}_{\mathrm{II}}\right)$, which is constitutive, is found upstream of the transcriptional activator encoded by eut $R$, providing a basal level of expression. EutR belongs to the AraC family of transcriptional regulators [Kofoid et al., 1999], and the presence of both its effectors, cobalamin and ethanolamine, is required for full induction of the operon to take place [Roof and Roth, 1992]. Due to the fact that it is encoded within the operon, EutR positively controls the induction of its own gene in a regulatory loop. This autoregulation may help to maintain induced expression by reducing competition between the EutBC ammonia lyase and EutR for the relatively low levels of cobalamin present in the cell [Garsin, 2010]. Conversely, the eut operons of Firmicutes are not regulated by EutR. Instead, transcription of the eut operon in these organisms is regulated via a two-component system encoded by eutVW [Fox et al., 2009]. Upon sensing ethanolamine, the histidine kinase EutW undergoes autophosphorylation, and 
Fig. 2. Self-assembly of BMC domain proteins into hexameric protein arrays. Monomers of BMC domain proteins, such as EutM (top left), assemble into homo-hexamers, with a central pore, as shown (bottom left). These hexamers form continuous layers (as shown on the right) and are believed to constitute the facets of the polyhedral BMC.

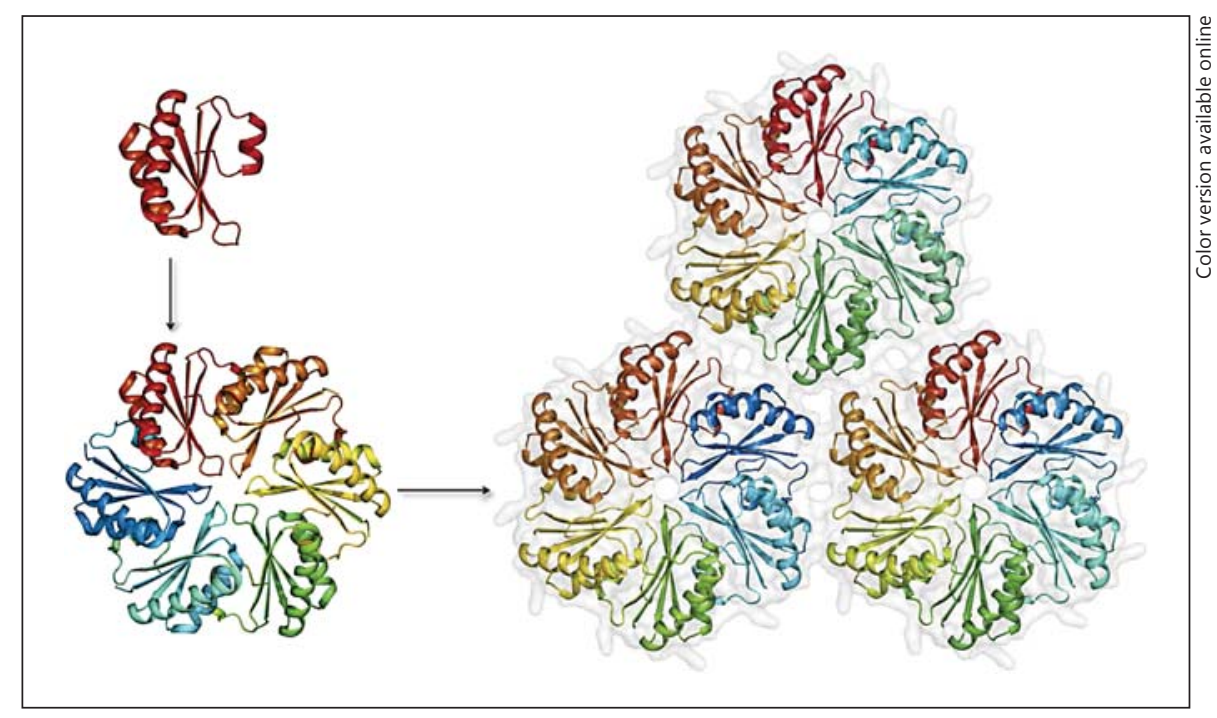

following phosphotransfer, the response regulator EutV is activated [Del Papa and Perego, 2008]. EutV, which has an ANTAR domain, likely binds RNA and disrupts transcriptional terminators, thereby upregulating gene expression. Several potential transcriptional terminator regions have been proposed at different sites within the operon. Additionally, a potential AdoCbl riboswitch has also been identified [Roth et al., 2007].

The existing models of the enzymatic pathways and modes of regulation of ethanolamine utilization provide us a partial insight into the role of Eut BMCs within the cell. Nevertheless, certain details are still missing and some hypotheses require experimental verification. We do not yet have a full understanding of the mechanism by which the shells self-assemble, or how the enzymes and their accessory components are encapsulated during this process. However, recent crystallographic studies into a number of the proteins that are encoded within the eut operon have provided key insights into the form and architecture of the Eut BMCs, serving to address these unanswered questions.

\section{Structural Aspects of Eut BMCs}

\section{Features of the Conserved BMC Domain}

As described earlier, one of the hallmarks of the proteins that form the outer shell of BMCs is that they share a common protein fold. The BMC domain, which is conserved across all types of BMC, typically consists of 90 amino acids, adopts an $\alpha / \beta$ barrel fold, and has a central four-stranded antiparallel sheet that is flanked by alpha helices, as exemplified by the crystal structure of EutM from E. coli and the EutM homologue CD1918 from C. difficile [Pitts et al., 2012; Takenoya et al., 2010; Tanaka et al., 2010]. Monomers of the BMC domain self-assemble to form cyclic hexamers; each hexamer represents a single tile in the regular mosaic pattern of each facet of the microcompartment shell (fig. 2). It is interesting to note that under different crystallization conditions, packing of EutM monomers within the crystal lattice is such that a 2D protein array of hexamers can be formed [Takenoya et al., 2010], suggesting a major role in self-assembly of the facets of the microcompartment shell. The properties of either side of the hexameric assembly are markedly different; one side can be described as concave, displaying an obvious depression, which is typically the side where the $\mathrm{N}$ - and C-termini are located. In the case of EutM, this concave side displays a highly charged surface, whereas its homologue CD1918 displays a more evenly distributed negative charge on both its concave and convex sides. These obvious charge distributions could be hypothesized to be involved in metabolite recognition, or perhaps in protein-protein interactions, although to date no evidence exists that could clarify the role that these charged areas play, or whether the concave or convex side of the hexamer faces towards the interior of the microcompartment. The $\mathrm{N}$ - and C-termini of various BMC domains tend to be of variable length and are flexible, and therefore often cannot be modeled in electron density maps. A narrow pore is located at the center of the hexamer; it has been hypothesized that the pores allow for diffusion of 
metabolites across the outer shell of the microcompartment [Tanaka et al., 2010].

\section{Variations of the BMC Domain}

Analysis of the crystal structures of apparent BMC domain proteins revealed several variations of this fold. Each of these variations has presumably developed over evolutionary time, thereby establishing the unique structural and functional aspects of the BMCs. One such variation is circular permutation of the BMC fold, such as that displayed by EutS, which shares a similar tertiary structure to EutM, but due to differences in the order of the underlying secondary structure of EutS, the N- and Ctermini are located on opposite sides of the hexamer [Tanaka et al., 2010]. The N-terminus of EutS extends above the surface of the hexamer, providing a barrelshaped pore that is lined by bulky hydrophobic residues. Also of interest is the bent hexamer formed by EutS from E. coli, which is unlike the flat hexameric assembly seen in the EutS homologue CD1908 from C. difficile [Pitts et al., 2012], as well as other BMC domain proteins. A single residue, Gly39, appears to be responsible for the bent arrangement of EutS in E. coli, which, when mutated to valine, causes a flattening of the hexamer assembly by almost $40^{\circ}$ [Tanaka et al., 2010]. It is not clear why EutS from $E$. coli would form a bent arrangement. Perhaps the bent conformation plays a role in the overall structure of some compartments, forming the edges of the outer shell, or allowing for a degree of flexibility or curvature in the shell. It has been shown that EutS from S. enterica is sufficient to form polyhedral bodies when expressed in $E$. coli, and upon expression of the mutation G39V, no recombinant BMCs are formed and functional targeting of enzymes to these bodies appears to be hindered [Choudhary et al., 2012]. These findings point to a diversity in sequence, structure and function in BMCs beyond our current knowledge and understanding, highlighting the need for further exploration.

Another departure from the canonical BMC fold is that of tandem BMC domains, in which case three monomers assemble as symmetric trimers, displaying pseudo 6-fold symmetry. One example of a tandem BMC domain is that of EutL, which is formed by two circularly permuted domains [Takenoya et al., 2010; Tanaka et al., 2010]. EutL has been crystallized in two distinct forms, with significant conformational changes that lead to an obvious opening and closing of the central pore, which in its open form has a radius of $11 \AA$. This variation of the BMC domain therefore appears to be somewhat flexible, and could represent a potential 'gated' mechanism by which specific large molecules are able to pass through the shell. In some cases, the BMC domain is fused to other protein domains. The crystal structure of the 60 residue C-terminal domain of EutK has been solved [Tanaka et al., 2010], revealing a helix-turn-helix domain, which suggests a role in binding to nucleic acids. However, the significance of this fusion is unknown; EutK is apparently a minor component of the Eut BMC shell, and unlike other BMC domain proteins, forms monomers in solution, which may point to a certain modularity in the formation of the outer shells by various BMC domain proteins.

Finally, not all of the proteins that form the shells of BMCs can be described as BMC domains. For example, EutN displays an OB fold, a domain which is usually associated with nucleic acid binding. It had originally been suggested that EutN might form a pentameric assembly, in a similar fashion to CcmL and CsoS4, which form the vertices of the icosahedral carboxysomes. However, the crystal structure of EutN expressed alone revealed a hexameric assembly [Tanaka et al., 2010]. It has been proposed that this assembly may not be physiologically relevant [Yeates et al., 2011]; however, this hypothesis does require verification. EutN paralogues from other microcompartment types and from other microorganisms have been crystallized as pentamers [Wheatley et al., 2013], and no evidence exists to suggest that EutN should behave any differently under crystallization conditions. Rather, this hexameric assembly could stem from the fact that Eut microcompartments, when viewed by traditional TEM techniques, appear to lack the icosahedral architecture associated with carboxysomes, suggesting a different mode in outer shell assembly. Indeed, recent studies have revealed that polyhedral structures can be formed by association of hexamers alone, both in the case of BMCs [Choudhary et al., 2012], and in other biological systems [Wright et al., 2007]. The mechanisms by which Eut microcompartments self-assemble are not yet understood due to the fact that there have been relatively few studies carried out in comparison to the well-studied carboxysomes and Pdu microcompartments; however, mounting evidence suggests a divergence from established models. Further studies are required to elucidate the role that the diverse evolutionary variants of the BMC domain play in the formation of Eut microcompartments.

\section{Passage of Molecules across the Outer Shell of BMCs}

The passage of the substrates and products involved in the Eut pathway, which is encapsulated within the Eut microcompartment, is believed to occur via the pores formed at the center of the hexameric BMC domain pro- 
Fig. 3. Comparison of the pore structure and charged surfaces of EutM and EutS from E. coli and C. difficile. Despite displaying similarities in hexameric assembly and pore size, the electrostatic properties of $\mathrm{BMC}$ domain protein homologues vary with host microorganism. Here, the surface properties of the hexameric assemblies of EutM and EutS from E. coli and C. difficile are displayed according to electrostatic potential, with red representing negative charge and blue representing positive charge.

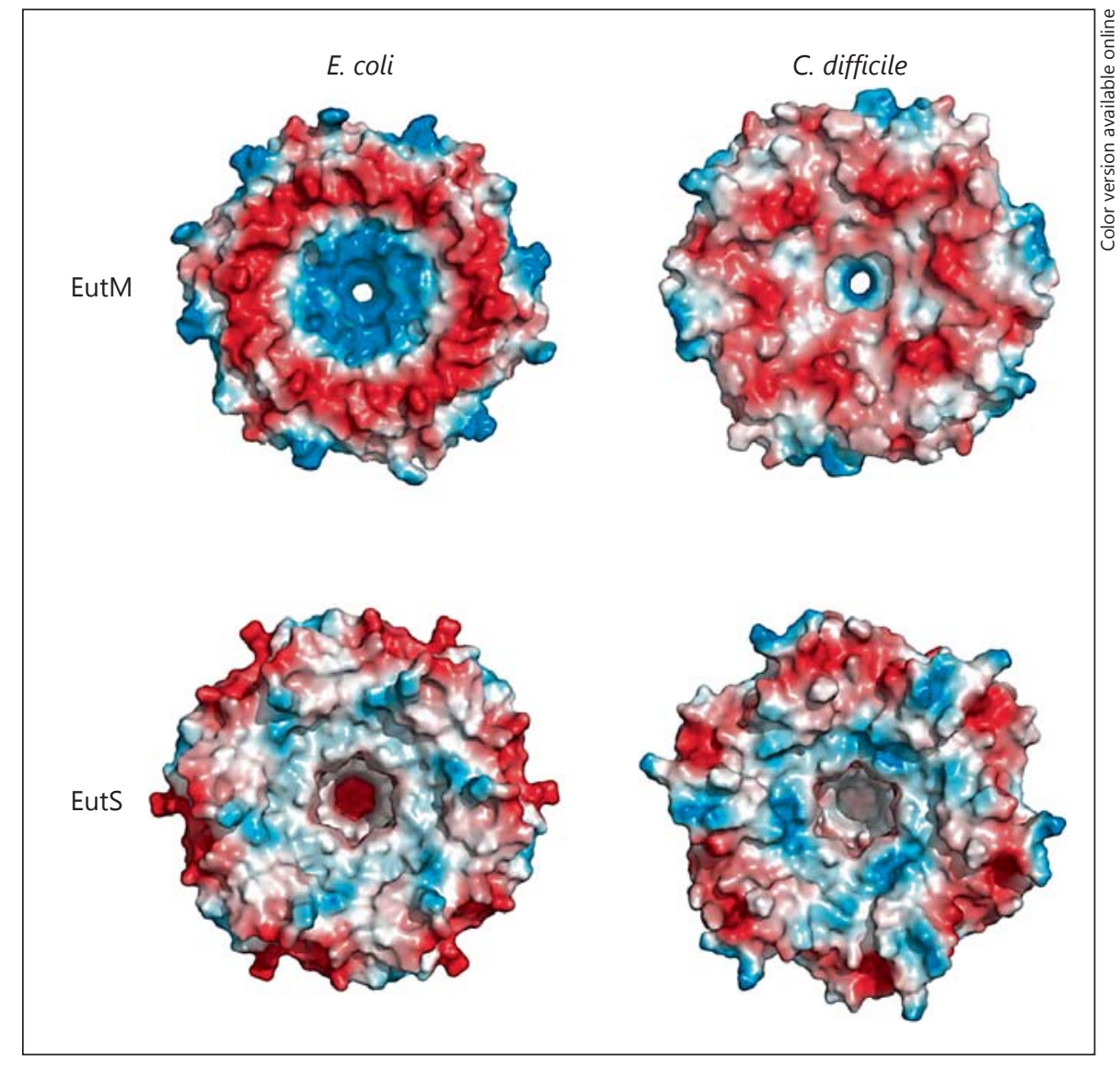

teins. The properties of these pores vary by size, charge and conformation, perhaps indicating different roles in function. EutS and CD1908 do not have a central open pore; rather, the N-termini of the EutS monomers forming the hexamer protrude from the surface as a $\beta$-barrel [Pitts et al., 2012; Tanaka et al., 2010]. The center of the $\beta$-barrel is blocked by the side chains of a glutamate residue, which appears to adopt alternate conformations in the crystal structures. Whether this flexible side chain can allow a gating mechanism to occur upon recognition of a particular molecule is not known. On the concave side of the hexamer, the depressed region surrounding the glutamate-blocked pore is negatively charged, with a diameter of $10 \AA$. The remainder of the opening is a conical cavity lined by hydrophobic residues, which at its widest point is approximately $25 \AA$ in diameter (fig. 3). Due to the occluded nature of this pore it is not clear what role it may play in the passage of substrates across the microcompartment shell.

EutM and CD1918 [Pitts et al., 2012; Takenoya et al., 2010; Tanaka et al., 2008] display a more open pore than that of EutS, with a diameter of $9 \AA$, and a positively charged pore surrounded by positively charged patches, which has been suggested to play a role in transport of the acetyl-phosphate product of Eut (fig. 3). EutN has a less circular pore, which is demarcated by depressions on either end of its longest axis, giving the region around the pore a 'slot-like' appearance. The pore is $12 \AA$ wide at its narrowest point, increasing to $23 \AA$ at the widest point of the concave side of the hexamer. The cavity is lined with positively charged patches and the face surrounding the base of the cavity forms a negatively charged ring; however, the pore itself does not display charged residues, making predictions in function futile.

As described previously, EutL has been crystallized in two distinct forms [Takenoya et al., 2010; Tanaka et al., 2010] and appears to be involved in a gated mechanism of transport across the shell. Due to the tandem nature of the BMC domain, the pore formed by EutL is triangular in shape; in its open conformation it has a width of $11 \AA$. Models have shown that this large pore size could facilitate passage of bulky molecules such as cobalamin or nu- 
cleotides, cofactors required for Eut. A gated mechanism would allow the uptake of these bulky cofactors only when required, preventing unnecessary passage of smaller substrates or loss of toxic intermediates such as acetaldehyde. While the crystal structures of the various Eut shell proteins have provided some clues with regards to the function of the pores in respect to transport of molecules across the shell, the hypotheses that have been put forward are not conclusive. Further ligand binding and mutagenesis studies will be required to fully understand the permeability and transport mechanisms of the microcompartment shells.

\section{Structural Insights into Nonshell Proteins Encoded on} the eut Operon

While intensive research has been devoted to elucidating the structures of the individual shell proteins of the Eut BMCs, few crystal structures have been solved for other, non-BMC domain proteins encoded within the eut operon. This discrepancy highlights the fact that Eut BMCs, having only recently been discovered, are relatively less well studied than the carboxysome and Pdu BMCs, and that we know far less about the form and function of Eut BMCs. To date, entries in the Protein DataBank exist for EutJ (unknown function) from Carboxydothermus hydrogenoformans, ethanolamine ammonia lyase light and heavy chains (EutBC complex) from E. coli and Listeria monocytogenes, a putative phosphate acetyltransferase (EutD) from E. coli, and EutQ (unknown function) from S. typhimurium and C. difficile. Of these, only the structural features of EutQ from $C$. difficile has been described in the literature [Pitts et al., 2012]. Intriguingly, despite displaying a cupin fold, EutQ lacks the catalytic histidine residues normally associated with metal binding, being replaced by acidic residues. Despite attempts to discover a possible ligand for this apparent active site, no catalytic function could be deduced. Hence, the role of EutQ in the Eut pathway remains unclear. Overall, our understanding of the Eut pathway members is lacking, and more extensive biochemical, biophysical and structural studies are required in order to fully understand the role and regulation of Eut BMCs in vivo.

\section{Localization of Enzymes to the Microcompartment Interior}

Targeting of Cargo Proteins to the Shell Interior

Whether evolved to metabolize ethanolamine, 1,2-propanediol, or fix carbon dioxide; it is clear that the lumen of intact BMCs is a busy locale, with a multitude of metabolic enzymes targeted therein. For example, Eut BMCs contain at least six metabolic components that are required for the conversion of ethanolamine to products destined for the tricarboxylic acid cycle including acetylphosphate, ethanol and acetyl-CoA [Kerfeld et al., 2010]. These include subunits of the characteristic ethanolamine ammonia lyase EutBC, along with the putative chaperonin EutA, an alcohol dehydrogenase EutG, and a cobalamin adenosyltransferase Eut T [Kofoid et al., 1999]. While not yet fully understood, the mechanism of how this targeting is achieved has been the focus of a number of recent reports and has obvious implications for future engineering of recombinant BMCs. Through bioinformatics research, a number of $\mathrm{N}$-terminal-targeting sequences of between 18 and 22 amino acids were recently identified in proteins predicted to reside within the BMC interior [Fan et al., 2010]. The requirement for these putative BMC signal sequences was confirmed by targeted deletions of the 10 or $14 \mathrm{~N}$-terminal residues of the propionaldehyde dehydrogenase (PduP) enzyme [Fan et al., 2010]. These mutations abolished the ability to target PduP to the BMC interior. Further proof corroborating the functionality of these signal sequences has been provided by a number of recent reports where fusions of these predicted $\mathrm{N}$-terminal extensions to reporter constructs, tags and functional enzymes such as $\beta$-galactosi-dase reliably targeted recombinant proteins to the BMC interior [Choudhary et al., 2012; Fan et al., 2010, 2012; Parsons et al., 2010]. Among the signal sequences predicted [Fan et al., 2010], two were identified specifically for Eut BMCs located at the N-termini of the EutC and EutG lumen proteins, respectively. Similarly for PduP, a 19-amino acid EutC signal sequence $\left(\right.$ EutC $\left._{1-19}\right)$ was recently shown to target EGFP and $\beta$-galactosidase to the Eut BMC lumen of recombinant shells formed in E. coli. This targeting was dependent upon the coexpression of either the EutS structural protein alone or with the entire suite (EutSMNLK) of predicted Eut shell proteins [Choudhary et al., 2012]. A number of parallel techniques unequivocally illustrated that the EutC $_{1-19}$-EGFP was indeed encapsulated within the recombinant compartments. Although EutG also contains an $\mathrm{N}$-terminal extension and was predicted to be an Eut BMC signal sequence [Fan et al., 2010], efforts to localize EGFP in a similar fashion to the $\mathrm{EutC}_{1-19}$ signal sequence failed [Choudhary et al., 2012]. It is not clear at this time why EutG does not contain a reliable BMC-targeting sequence in spite of the presence of an $\mathrm{N}$-terminal extension typical of other targeted cargo 
[Choudhary et al., 2012; Fan et al., 2010], although it is possible that a longer signal sequence might be required to increase stability and improve recognition. Indeed, the Ras-like GTPase enzyme PduV also targets EGFP to recombinant Pdu compartments in E. coli, but targeting was more efficient with a longer 98 -amino-acid tag as compared to a shorter 42-amino-acid version [Parsons et al., 2010].

The identification of these BMC-targeting sequences has begun to open a toolkit of sorts, which provides researchers with the means to engineer targeted biochemistry within recombinant compartments in the very near future. These advances also allow groups to delve into many interesting questions relating to the fundamental science of BMC assembly and their context within the cellular environment. As described earlier, it is well established that the assembly of functional BMCs in vivo requires a number of stable and highly evolved interactions between numerous shell proteins. These interactions not only serve to sort repeated units of shell monomers into intricate lattices and repeated facets which yield the elegant and complex surface of these shells [Yeates et al., 2011], but are also predicted to be required for the encapsulation of cargo proteins during shell assembly. This proposal of interaction between cargo and shell proteins has been posed several times in recent years, and has since been functionally confirmed by a number of reports. For the EutC-targeting sequence, successful localization of EGFP to the BMC lumen was dependent upon the coexpression of the structural protein EutS, providing a connection between formation of Eut compartments and encapsulation of cargo proteins. Indeed, EutS is unique among BMC structural components as it alone is sufficient for recombinant production of viable, intact Eut BMC shells in E. coli [Choudhary et al., 2012]. Contrastingly, Pdu BMCs require the coexpression of multiple shell proteins to produce a recombinant structure in vivo [Parsons et al., 2010a]. EutS is also necessary for BMC formation; although five separate shell proteins are predicted to contribute to the compartment shell, combinations of different shell proteins lacking EutS failed to produce functional compartments in E. coli [Choudhary et al., 2012a]. Taken together, these results indicate that EutS likely recruits cargo proteins to the $\mathrm{BMC}$ interior via interaction with an $\mathrm{N}$ terminal signal sequence and that it also likely interacts directly with the N-terminus of EutC [Choudhary et al., 2012].
Mechanistic Aspects of Cargo Protein Encapsulation

Although a convincing amount of circumstantial evidence had been collected which indicated that cargo proteins were targeted to the BMC lumen via some sort of interaction with shell proteins, it was not until very recently that this assumption was clearly illustrated. Fan et al. [2012] used a number of in silico and wet techniques to confirm that the N-terminal region of a Pdu cargo protein, PduP, interacted with the C-terminus of a Pdu shell monomer, PduA, a homologue of the structural protein EutM. This provided the first functional proof of how the PduP cargo was being encapsulated within the lumen of the assembling BMC. Structural analyses and targeted mutagenesis of PduP and PduA indicated that this interaction involves conserved helices at the $\mathrm{N}$ - and $\mathrm{C}$-termini of the cargo and shell protein, respectively. On both of these conserved helices, residues critical for the proposed interaction were identified. For the cargo protein, substitution of Glu7, Ile10 and/or Leu14 with alanine significantly impaired purification of PduP and the isolation of active PduP from BMC preparations [Fan et al., 2012]. These residues coincidently all protrude from one side of this short helix in a nearly perfect conformation for tight interaction with the $\mathrm{C}$-terminus of PduA. Combined with a reciprocal set of residues from the $\mathrm{C}$-terminus of the PduA shell protein, researchers now had identified a pair of short, conserved motifs which are required for the encapsulation of cargo proteins and provided functional support for the earlier claim regarding the mechanism of shell and cargo protein interaction during BMC biogenesis. Although some variation exists, these same conserved motifs can be readily identified from a number of cargo proteins and shell monomers, indicating that this may be a common mechanism.

\section{Different Means to the Same End}

In spite of the unquestionable support for an encapsulation-based dogma for targeting of cargo proteins destined for the BMC interior, a number of recent reports indicate that several options may exist to achieve this same goal. Firstly, and most obviously, a number of enzymes that are known to be associated with BMCs lack N-terminal-targeting sequences; how then do they achieve localization? This is indeed true for many metabolic enzymes of the Eut BMC, and also the Pdu compartments and the carboxysomes [Fan et al., 2010]. From this precipice, two plausible options exist; either there are variations in the composition of sequences that function as BMC-targeting sequences beyond those identified so far, or there are other, perhaps more complex mecha-

J Mol Microbiol Biotechnol 2013;23:308-320 
nisms for recruiting enzymes to the BMC. As it turns out, both might actually be true. Firstly, variation does indeed exist in the sequences which target cargo to the BMC beyond the canonical example described above. Brief bioinformatics analyses have revealed a number of variations and permutations in the $\mathrm{N}$ - and C-termini of both cargo and shell proteins, respectively [Fan et al., 2012]. Secondly, there is evidence for more complex interactions and recruitment mechanisms for various BMC cargo enzymes, which indicate that these proteins might interact with more than one partner and/or aid in the recruitment of each other and accessory proteins. For example, the carboxysome microcompartment protein $\mathrm{CcmM}$ has been shown to form complexes with $\mathrm{CcaA}$ and Rubisco and may act to cross-link these components together and contribute to carboxysome assembly [Long et al., 2007]. Moreover, $\mathrm{CcmM}$ and $\mathrm{CcmN}$ are known to function as part of a bicarbonate dehydration complex or BDC, which is believed to aid in the recruitment of other metabolic enzymes and is known to interact with structural shell proteins $\mathrm{CcmK}$ and CcmL [Cot et al., 2008]. Thus, a myriad of sophisticated avenues exist for the targeting of cargo proteins to the BMC interior and it is likely that several of these are employed simultaneously as BMC biogenesis progresses in vivo.

\section{Phylogeny of Eut Compartments}

\section{Variation on a Conserved Theme}

As mentioned previously, early reports of BMCs date back nearly 6 decades now and initiated a still ongoing explosion of research into the diversity of BMC form and function that covers both applied engineering approaches and more fundamental research topics. Moreover, the discovery of these intracellular compartments began to shed light onto the sophisticated nature of the prokaryotic intracellular environment; a field which now contributes a staggering number of high-impact articles yearly. Since that time, a great deal of variation in BMC types has been uncovered, with some still to be characterized. Beyond the well-developed Eut, Pdu and carboxysome compartments, more obscure and intriguing examples exist such as the metal-crystallizing compartments of magnetotrophic bacteria [Komeili, 2012]. BMCs with newly discovered metabolic variations have also recently been identified, including those which breakdown 1,2-propanediol via a glycyl radical (Grp) and others possibly involved in the metabolism of amino alcohol groups [Jorda et al., 2013]. Although distinct, it is clear that the ge- netic building blocks for these new BMC types were coopted from already existing components. Like their predecessors, the shells of these newly described BMCs are comprised of several structural proteins homologous to previously well-characterized examples from Eut, Pdu and carboxysome compartments [Jorda et al., 2013]. The Grp BMC also appears to contain cargo proteins which are likely targeted to the shell interior via $\mathrm{N}$-terminal signal sequences in a manner similar to that described earlier in this review.

Although sporadically conserved, the number of prokaryotic genomes containing BMC fold-containing proteins is staggering. Currently, a brief bioinformatics survey of prokaryotic genomes for proteins containing a BMC domain will yield greater than 4,700 sequences, from well over 500 species. These hits are distributed over twelve phyla within the Eubacteria, yet examples from the Archaea and Eukaryota are entirely absent. Numerous enterobacterial genera within the Proteobacteria contain Eut operons such as Salmonella, Escherichia, Klebsiella, Clostridium, Shigella, Yersinia and Proteus, to name a few. Eut BMCs have likely been selected for over eons in these genera as ethanolamine is a major component of mammalian cell membranes, and thus it makes perfect sense that a pathogenic bacterium residing within the digestive system of a mammalian host would evolve a specialized metabolic reaction center to provide a unique energy source [Kendall et al., 2012]. In many cases, these same genera have the ability to produce Pdu compartments as well. BMC domain proteins among these phyla are easily identifiable and, for the most part, very well conserved. Duplication of BMC-containing genomic regions is also quite rampant, with multiple paralogues of BMC proteins often found within a single genome. Indeed, a recent study identified the BMC domain as the most prolific protein family amongst those in which multiple paralogues are often found in a single operon [Beeby et al., 2009].

Interestingly, largely uncharacterized examples also exist, such as the aptly-named Bacillus megaterium. This Gram-positive bacteria of the phyla Firmicutes can grow to micrometer sizes and has been successfully applied in the production of vitamin B12 precursors [Vary et al., 2007] and starch-modifying enzymes such as $\alpha$ - and $\beta$-amylases [Panbangred et al., 2000]. Although little to no data exist regarding BMC form and function in this unique microbe, there is clearly a vitamin B12-dependant Eut operon, which is nearly identical to the canonical examples from $E$. coli and S. enterica. One notable open reading frame lacking from the $B$. megaterium Eut oper- 
on is a bicupin domain protein homologous to EutQ, which is typically found proximal to EutP and EutS homologues. Although BLAST searches against B. megaterium with the $S$. enterica EutQ sequence does yield some hits, their reliability is tenuous at best, having low scores and being located megabases away from the rest of the operon. Considering the well-established propensity for components of Eut operons to maintain proximity to each other, it seems unlikely that a legitimate homologue of the bicupin domain protein EutQ actually exists in the B. megaterium genome. The transcriptional regulation of this and many other Gram-positive Eut operons is controlled by the EutVW two-component system [Eppinger et al., 2011] discussed earlier in this review, which is mutually exclusive with the EutR element more commonly found in Gram-negative enterobacteria.

\section{Evolutionary History of BMCs}

For quite some time, the evolution of BMCs has remained mysterious. At first glance, their architecture bears a striking resemblance to viral capsids (fig. 1). However, BMCs do not contain nucleic acids. Rather, the BMC lumen contains a diverse array of metabolic enzymes which allow their hosts to occupy a unique ecological niche (metabolism of cytotoxic compounds, fixation of carbon dioxide, access to unique carbon and nitrogen sources, etc.). Viral capsids are instead linked to the evolution of a different compartment type. The encapsulin or linocin-like proteins found in numerous bacterial phyla are involved in assembling the smallest known protein-based microcompartment, with a diameter of only $25 \mathrm{~nm}$ as compared to upwards of $150 \mathrm{~nm}$ for Eut BMCs [Sutter et al., 2008]. These compartments are icosahedral shells and have clearly evolved from viral origin. The mechanism for targeting cargo proteins to these compartments is also different. Unlike Eut BMCs (and also Pdu compartments), the cargo is encapsulated as the shell is forming by means of a direct, Cterminal fusion to the shell protein [Corchero and Cedano, 2011].

From an evolutionary perspective, BMCs have a very interesting history. Firstly, all of the well-characterized BMC types arose from as-of-yet unknown origins, with the only tenuous and really unsupported link being increased complexity in form and function of encapsulins. Subsequently, selective pressures such as changes in atmospheric $\mathrm{CO}_{2}$ levels and the microenvironment of the mammalian gastrointestinal tract likely lead to the expansion of BMC types in numerous prokaryotic lineages. Lastly, virtually all BMC types were lost in the evolution of eukaryotic organisms and, indeed, numerous studies have rightfully stated that no examples of BMC protein homologues exist in the Archaea or Eukaryota. Why this is still remains in question, but it seems likely that a number of variables including an increase in cell types, compartmentalization, sequestration and trafficking achieved by other means contributed to their absence in more derived, multicellular organisms. From a metabolic perspective, it also seems obvious that an organism would forfeit the production of these specialized and costly structures without strong, selective pressure to maintain them.

The plastids of all eukaryotes within the Plantae kingdom are believed to have derived from a single, endosymbiotic event over 1.5 billion years ago. The lineage of these plastids can be traced back to cyanobacterial origins. As these endosymbionts persisted within their host, their genomes underwent drastic reduction, maintaining only components valuable to the host (such as photosynthetic machinery) and becoming entirely dependent upon the host. The entire complement of derived plastids found in extant plants evolved from this singular event. However, a second and much more recent endosymbiotic event was described involving a cyanobacterial symbiont which persisted in the photosynthetic amoebae Paulinella chromatophora [Nowack et al., 2008]. Not surprisingly, the genome of the P. chromatophora endosymbiont has contracted drastically and recent studies indicate that a small amount of genetic material ( $<1 \%$ as compared to $10-15 \%$ in plants) has been transferred from the endosymbiont to the host genome [Bhattacharya et al., 2012; Nowack et al., 2011]. As is the case for the primary endosymbiotic event, it is clear that the chromatophores in this strange creature are $\alpha$-cyanobacterial in origin, having been derived from organisms known to contain carboxysomes (i.e. from genera such as Prochlorococcus). This finding becomes quite interesting in regards to the evolution of BMCs. A BLAST search for potential Eut shell homologues against the available $P$. chromatophora databases indicates that the chromatophore contains an EutM homologue, thus providing a link between prokaryotic BMCs and primary plastid evolution in eukaryotes. Taken together, the evolutionary history of BMCs tells a story which begins with vague beginnings, followed by rampant expansion of form and function within prokaryotic organisms, and lastly punctuated by a severe contraction largely as a result of a lack of selective pressure in derived, multicellular organisms. 


\section{Future Prospects}

A great deal of information has been accrued over a very short period of time regarding the form and function of BMCs. Structural and functional properties of native and recombinant Eut compartments have greatly expanded our knowledge base and laid the groundwork for future experiments which truly push the boundaries of synthetic biology. For instance, it is not clear how all of the metabolic enzymes targeted to the BMC lumen actually get there. It is likely based on recent reports that several different encapsulation strategies are utilized in conjunction with the scheme outlined by Fan et al. [2012a]. Once inside the BMC, it seems unlikely that all lumen-targeted proteins would remain affixed to the interior wall of the $\mathrm{BMC}$. If that were the case, only a fraction of the interior space would be occupied. The obvious question here then becomes, why create such a costly and massive structure without efficiently utilizing the entire lumen area? One would expect that from an evolutionary perspective, the ability to maximize space utilization and therefore metabolic flux and reaction efficiency would be strongly favored over generations. Moreover, the maintenance of the shell architecture is likely stabilized and supported by the presence of lumen-associated proteins of various types in a manner similar to the turgor pressure which affords rigidity to plant cells. It seems plausible then that some sort of cleavage mechanism might exist to liberate enzymes from their interaction with shell proteins and allow them to diffuse throughout the luminal space. This mechanism might be active, proteolytic cleavage of recognition sequences on lumen cargo, or simply that the established interaction between cargo proteins and shells is not a committed, but rather transient interaction.

From a purely fundamental perspective, there remain many questions regarding what controls the number of Eut BMCs formed in vivo. In the native organism S. enterica, Eut BMCs are numerous and mobile in vivo and yet, recombinant compartments expressed in E. coli only form at a rate of one per cell and are immobile [Choudhary et al., 2012b]. This type of dynamic mobility has not been observed for Pdu compartments which are also formed by S. enterica; however, recent studies have suggested that compartments might be linked to cellular machinery to control localization and movement [Parsons et al., 2010]. The ability to control cellular locale, mobility and number of reaction centers in vivo has astounding implications for the eventual engineering of these compartments for truly designer pathway construction. These are truly tantalizing research areas with much left to be discovered.

\section{Acknowledgements}

Research on bacterial microcompartments in the corresponding author's laboratory is supported by the Institute on the Environment, Biocatalysis Initiative and the BioTechnology Institute (University of Minnesota), and the Office of Naval Research (grant No. N00014-10-1-0157).

\section{References}

Beeby M, Bobik TA, Yeates TO: Exploiting genomic patterns to discover new supramolecular protein assemblies. Protein Sci 2009;18: 69-79.

Bhattacharya D, Price DC, Yoon HS, Yang EC, Poulton NJ, Andersen RA, Das SP: Single cell genome analysis supports a link between phagotrophy and primary plastid endosymbiosis. Sci Rep 2012;2:356.

Bobik TA: Polyhedral organelles compartmenting bacterial metabolic processes. Appl Microbiol Biotech 2006;70:517-525.

- Bobik TA, Havemann GD, Busch RJ, Williams DS, Aldrich HC: The propanediol utilization $(p d u)$ operon of Salmonella enterica serovar Typhimurium LT2 includes genes necessary for formation of polyhedral organelles involved in coenzyme $\mathrm{B}_{12}$-dependent 1,2-propanediol degradation. J Bac 1999;181:59675975.
Brinsmade SR, Escalante-Semerena JC: The eutD gene of Salmonella enterica encodes a protein with phosphotransacetylase enzyme activity. J Bac 2004;186:1890-1892.

Buan NR, Suh SJ, Escalante-Semerena JC: The eutT gene of Salmonella enterica encodes an oxygenlabile, metal-containing ATP:corrinoid adenosyltransferase enzyme. J Bac 2004;186:5708-5714.

-Cannon GC, Bradburne CE, Aldrich HC, Baker SH, Heinhorst S, Shively JM: Microcompartments in prokaryotes: carboxysomes and related polyhedra. Appl Environ Microbiol 2001;67:5351-5361.

Cannon GC, English RS, Shively JM: In situ assay of ribulose-1,5-bisphosphate carboxylase/oxygenase in Thiobacillus neapolitanus. J Bac 1991;173:1565-1568.

Chen P, Andersson DI, Roth JR: The control region of the pdu/cob regulon in Salmonella typhimurium. J Bac 1994;176:5474-5482.
Cheng S, Liu Y, Crowley CS, Yeates TO, Bobik TA: Bacterial microcompartments: their properties and paradoxes. Bioessays 2008;30:1084-1095.

-Choudhary S, Quin MB, Sanders MA, Johnson ET, Schmidt-Dannert C: Engineered protein nano-compartments for targeted enzyme localization. PLoS One 2012;7:e33342.

Corchero JL, Cedano J: Self-assembling, proteinbased intracellular bacterial organelles: emerging vehicles for encapsulating, targeting and delivering therapeutical cargos. Microb Cell Fact 2011;10:8

-Cot SS, So AK, Espie GS: A multiprotein bicarbonate dehydration complex essential to carboxysome function in cyanobacteria. J Bac 2008; 190:936-945.

Crowley CS, Cascio D, Sawaya MR, Kopstein JS, Bobik TA, Yeates TO: Structural insight into the mechanisms of transport across the Salmonella enterica Pdu microcompartment shell. J Biol Chem 2010;285:37838-37846. 
Crowley CS, Sawaya MR, Bobik TA, Yeates TO: Structure of the PduU shell protein from the Pdu microcompartment of Salmonella. Structure 2008;16:1324-1332.

-Del Papa MF, Perego M: Ethanolamine activates a sensor histidine kinase regulating its utilization in Enterococcus faecalis. J Bac 2008;190: 7147-7156.

Drews G, Niklowitz W: Cytology of Cyanophycea. II. Centroplasm and granular inclusions of Phormidium uncinatum. Arch Microbiol 1956;24:147-162.

Eppinger M, Bunk B, Johns MA, Edirisinghe JN, Kutumbaka KK, Koenig SS, Creasy HH, Rosovitz MJ, Riley DR, Daugherty S, Martin M, Elbourne LD, Paulsen I, Biedendieck R, Braun C, Grayburn S, Dhingra S, Lukyanchuk V, Ball B, Ul-Qamar R, Seibel J, Bremer E, Jahn D, Ravel J, Vary PS: Genome sequences of the biotechnologically important Bacillus megaterium strains QM B1551 and DSM319. J Bac 2011;193:4199-4213.

-Fan C, Cheng S, Liu Y, Escobar CM, Crowley CS, Jefferson RE, Yeates TO, Bobik TA: Short Nterminal sequences package proteins into bacterial microcompartments. Proc Natl Acad Sci USA 2010;107:7509-7514.

- Fan C, Cheng S, Sinha S, Bobik TA: Interactions between the termini of lumen enzymes and shell proteins mediate enzyme encapsulation into bacterial microcompartments. Proc Natl Acad Sci USA 2012;109:14995-15000.

-Fox KA, Ramesh A, Stearns JE, Bourgogne A, Reyes-Jara A, Winkler WC, Garsin DA: Multiple posttranscriptional regulatory mechanisms partner to control ethanolamine utilization in Enterococcus faecalis. Proc Natl Acad Sci USA 2009;106:4435-4440.

Garsin DA: Ethanolamine utilization in bacterial pathogens: roles and regulation. Nat Rev Microbiol 2010;8:290-295.

Gitai Z: The new bacterial cell biology: moving parts and subcellular architecture. Cell 2005; 120:577-586

Havemann GD, Bobik TA: Protein content of polyhedral organelles involved in coenzyme B12-dependent degradation of 1,2-propanediol in Salmonella enterica serovar Typhimurium LT2. J Bac 2003;185:5086-5095.

-Heldt D, Frank S, Seyedarabi A, Ladikis D, Parsons JB, Warren MJ, Pickersgill RW: Structure of a trimeric bacterial microcompartment shell protein, EtuB, associated with ethanol utilization in Clostridium kluyveri. Biochem J 2009;423:199-207.

-Jorda J, Lopez D, Wheatley NM, Yeates TO: Using comparative genomics to uncover new kinds of protein-based metabolic organelles in bacteria. Protein Sci 2013;22:179-195.

Kendall MM, Gruber CC, Parker CT, Sperandio $\mathrm{V}$ : Ethanolamine controls expression of genes encoding components involved in interkingdom signaling and virulence in enterohemorrhagic Escherichia coli O157:H7. MBio 2012; 3:e00050-12.
Kerfeld CA, Heinhorst S, Cannon GC: Bacterial microcompartments. Annu Rev Microbiol 2010;64:391-408.

Kerfeld CA, Sawaya MR, Tanaka S, Nguyen CV, Phillips M, Beeby M, Yeates TO: Protein structures forming the shell of primitive bacterial organelles. Science 2005;309:936-938.

-Klein MG, Zwart P, Bagby SC, Cai F, Chisholm SW, Heinhorst S, Cannon GC, Kerfeld CA: Identification and structural analysis of a novel carboxysome shell protein with implications for metabolite transport. J Mol Biol 2009;392:319-333.

Kofoid E, Rappleye C, Stojiljkovic I, Roth J: The 17-gene ethanolamine (eut) operon of Salmonella typhimurium encodes five homologues of carboxysome shell proteins. J Bac 1999;181: 5317-5329.

Komeili A: Molecular mechanisms of compartmentalization and biomineralization in magnetotactic bacteria. FEMS Microbiol Rev 2012;36:232-255.

Long BM, Badger MR, Whitney SM, Price GD: Analysis of carboxysomes from Synechococcus PCC7942 reveals multiple Rubisco complexes with carboxysomal proteins CcmM and CcaA. J Biol Chem 2007;282:2932329335.

Mori K, Bando R, Hieda N, Toraya T: Identification of a reactivating factor for adenosylcobalamin-dependent ethanolamine ammonia lyase. J Bac 2004;186:6845-6854.

Nowack ECM, Melkonian M, Gloeckner G: Chromatophore genome sequence of Paulinella sheds light on acquisition of photosynthesis by eukaryotes. Curr Biol 2008;18:410-418.

Nowack ECM, Vogel H, Groth M, Grossman AR, Melkonian M, Glockner G: Endosymbiotic gene transfer and transcriptional regulation of transferred genes in Paulinella chromatophora. Mol Biol Evol 2011;28:407-422.

Panbangred W, Weeradechapon K, Udomvaraphant S, Fujiyama K, Meevootisom V: High expression of the penicillin $\mathrm{G}$ acylase gene (pac) from Bacillus megaterium UN1 in its own pac minus mutant. J Appl Microbiol 2000;89:152-157.

- Parsons JB, Frank S, Bhella D, Liang M, Prentice MB, Mulvihill DP, Warren MJ: Synthesis of empty bacterial microcompartments, directed organelle protein incorporation, and evidence of filament-associated organelle movement. Mol Cell 2010;38:305-315.

Penrod JT, Mace CC, Roth JR: A pH-sensitive function and phenotype: evidence that EutH facilitates diffusion of uncharged ethanolamine in Salmonella enterica. J Bac 2004;186: 6885-6890.

Penrod JT, Roth JR: Conserving a volatile metabolite: a role for carboxysome-like organelles in Salmonella enterica. J Bac 2006; 188:28652874.

Pitts AC, Tuck LR, Faulds-Pain A, Lewis RJ, Marles-Wright J: Structural insight into the Clostridium difficile ethanolamine utilization microcompartment. PLoS One 2012;7:e48360.
Roof DM, Roth JR: Ethanolamine utilization in Salmonella typhimurium. J Bac 1988;170: 3855-3863.

Roof DM, Roth JR: Functions required for vitamin B12-dependent ethanolamine utilization in Salmonella typhimurium. J Bac 1989;171: 3316-3323.

Roof DM, Roth JR: Autogenous regulation of ethanolamine utilization by a transcriptional activator of the eut operon in Salmonella typhimurium. J Bac 1992;174:6634-6643.

Roth A, Winkler WC, Regulski EE, Lee BW, Lim J, Jona I, Barrick JE, Ritwik A, Kim JN, Welz R, Iwata-Reuyl D, Breaker RR: A riboswitch selective for the queuosine precursor preQ1 contains an unusually small aptamer domain. Nat Struct Mol Biol 2007;14:308-317.

Schmid MF, Paredes AM, Khant HA, Soyer F, Aldrich HC, Chiu W, Shively JM: Structure of Halothiobacillus neapolitanus carboxysomes by cryo-electron tomography. J Mol Biol 2006;364:526-535.

Sheppard DE, Penrod JT, Bobik T, Kofoid E, Roth JR: Evidence that a B12-adenosyl transferase is encoded within the ethanolamine operon of Salmonella enterica. J Bac 2004;186:76357644.

- Shively JM, Ball F, Brown DH, Saunders RE: Functional organelles in prokaryotes: polyhedral inclusions (carboxysomes) of Thiobacillus neapolitanus. Science 1973b;182:584586.

Shively JM, Ball FL, Kline BW: Electron microscopy of the carboxysomes (polyhedral bodies) of Thiobacillus neapolitanus. J Bac 1973a;116: 1405-1411.

Stojiljkovic I, Baumler AJ, Heffron F: Ethanolamine utilization in Salmonella typhimurium: nucleotide sequence, protein expression, and mutational analysis of the cchA cchB eutE eutJ eut $G$ eut $H$ gene cluster. J Bac 1995;177:13571366.

Sutter M, Boehringer D, Gutmann S, Guenther S, Prangishvili D, Loessner MJ, Stetter KO, Weber-Ban E, Ban N: Structural basis of enzyme encapsulation into a bacterial nanocompartment. Nat Struct Mol Biol 2008;15: 939-947.

Takenoya M, Nikolakakis K, Sagermann M: Crystallographic insights into the pore structures and mechanisms of the EutL and EutM shell proteins of the ethanolamine-utilizing microcompartment of Escherichia coli. J Bac 2010; 192:6056-6063.

Tanaka S, Kerfeld CA, Sawaya MR, Cai F, Heinhorst S, Cannon GC, Yeates TO: Atomic-level models of the bacterial carboxysome shell. Science 2008;319:1083-1086.

Tanaka S, Sawaya MR, Phillips M, Yeates TO: Insights from multiple structures of the shell proteins from the beta-carboxysome. Protein Sci 2009; 18:108-120.

Tanaka S, Sawaya MR, Yeates TO: Structure and mechanisms of a protein-based organelle in Escherichia coli. Science 2010;327:81-84. 
Tsai Y, Sawaya MR, Cannon GC, Cai F, Williams EB, Heinhorst S, Kerfeld CA, Yeates TO: Structural analysis of CsoS1A and the protein shell of the Halothiobacillus neapolitanus carboxysome. PLoS Biol 2007;5:e144.

Tsoy O, Ravcheev D, Mushegian A: Comparative genomics of ethanolamine utilization. J Bac 2009;191:7157-7164.

-Vary PS, Biedendieck R, Fuerch T, Meinhardt F, Rohde M, Deckwer W-D, Jahn D: Bacillus megaterium from simple soil bacterium to industrial protein production host. Appl Microbiol Biotech 2007;76:957-967.
Wheatley NM, Gidaniyan SD, Liu Y, Cascio D, Yeates TO: Bacterial microcompartment shells of diverse functional types possess pentameric vertex proteins. Protein Sci 2013;22: 660-665.

Wright ER, Schooler JB, Ding HJ, Kieffer C, Fillmore C, Sundquist WI, Jensen GJ: Electron cryotomography of immature HIV-1 virions reveals the structure of the CA and SP1 Gag shells. EMBO J 2007;26:2218-2226. eates TO, Crowley CS, Tanaka S: Bacterial microcompartment organelles: protein shell structure and evolution. Annu Rev Biophys 2010;39:185-205.

-Yeates TO, Kerfeld CA, Heinhorst S, Cannon GC, Shively JM: Protein-based organelles in bacteria: carboxysomes and related microcompartments. Nat Rev Microbiol 2008;6: 681-691.

Yeates TO, Thompson MC, Bobik TA: The protein shells of bacterial microcompartment organelles. Curr Opin Struct Biol 2011;21:223231 